PROCEEDINGS OF THE

AMERICAN MATHEMATICAL SOCIETY

Volume 129, Number 4, Pages 1021-1029

S 0002-9939(00)05714-2

Article electronically published on October 11, 2000

\title{
SINGLE ELEMENTS OF FINITE CSL ALGEBRAS
}

\author{
W. E. LONGSTAFF AND ORESTE PANAIA
}

(Communicated by David R. Larson)

\begin{abstract}
An element $s$ of an (abstract) algebra $\mathcal{A}$ is a single element of $\mathcal{A}$ if $a s b=0$ and $a, b \in \mathcal{A}$ imply that $a s=0$ or $s b=0$. Let $X$ be a real or complex reflexive Banach space, and let $\mathcal{B}$ be a finite atomic Boolean subspace lattice on $X$, with the property that the vector sum $K+L$ is closed, for every $K, L \in \mathcal{B}$. For any subspace lattice $\mathcal{D} \subseteq \mathcal{B}$ the single elements of $A \lg \mathcal{D}$ are characterised in terms of a coordinatisation of $\mathcal{D}$ involving $\mathcal{B}$. (On separable complex Hilbert space the finite distributive subspace lattices $\mathcal{D}$ which arise in this way are precisely those which are similar to finite commutative subspace lattices. Every distributive subspace lattice on complex, finite-dimensional Hilbert space is of this type.) The result uses a characterisation of the single elements of matrix incidence algebras, recently obtained by the authors.
\end{abstract}

\section{INTRODUCTION AND PRELIMINARIES}

Throughout, $X$ will denote a non-zero real or complex reflexive Banach space, and $H$ will denote a non-zero complex separable Hilbert space. Also, $\mathbb{F}$ will denote the real or complex field. By a subspace of $X$ we mean a norm-closed linear manifold and by an operator on $X$ we mean a bounded linear transformation acting on $X$. The set of operators on $X$ is denoted by $\mathcal{B}(X)$. By a subspace lattice on $X$ we mean a family $\mathcal{L}$ of subspaces of $X$ satisfying (i) (0), $X \in \mathcal{L}$ and (ii) for every family $\left\{L_{\gamma}\right\}_{\Gamma}$ of elements of $\mathcal{L}, \cap_{\Gamma} L_{\gamma} \in \mathcal{L}, \vee_{\Gamma} L_{\gamma} \in \mathcal{L}$ (where ' $\vee$ ' denotes 'closed linear span'). A subspace lattice on a Hilbert space is commutative if the (orthogonal) projections onto any two of its members commute. (As usual $P_{L}$ will denote the (orthogonal) projection onto the subspace L.) The abbreviation 'CSL' will be used for 'commutative subspace lattice'. A subspace lattice on $X$ is Boolean if it is complemented and distributive, and atomic if each of its elements is the (closed linear) span of the atoms that it contains. The abbreviation 'ABSL' will be used for 'atomic Boolean subspace lattice'. In any subspace lattice $\mathcal{L}$ the 'minus operation' is the self-map defined by

$$
L_{-}=\bigvee\{M \in \mathcal{L}: L \nsubseteq M\},
$$

for any element $L \in \mathcal{L}$ (so that $L \nsubseteq M \Rightarrow M \subseteq L_{-}$). The annihilator $\mathcal{S}^{\perp}$ of a subset $\mathcal{S} \subseteq X$ is, as usual, given by $\mathcal{S}^{\perp}=\left\{x^{*} \in X^{*}: x^{*}(x)=0\right.$, for every $\left.x \in \mathcal{S}\right\}$, where $X^{*}$ denotes the topological dual of $X$. For any vectors $f \in X, e^{*} \in X^{*}$ the

Received by the editors June 20, 1999.

2000 Mathematics Subject Classification. Primary 47L35; Secondary 47C05.

(C)2000 American Mathematical Society 
operator $e^{*} \otimes f \in \mathcal{B}(X)$ is defined by $e^{*} \otimes f(x)=e^{*}(x) f$, for every $x \in X$. For any family $\mathcal{L}$ of subspaces of $X, \operatorname{Alg} \mathcal{L}$ is the operator algebra given by

$$
\operatorname{Alg} \mathcal{L}=\{T \in \mathcal{B}(X): T(L) \subseteq L, \text { for every } L \in \mathcal{L}\}
$$

The results from abstract lattice theory that we use are to be found in [2].

Let $n \in \mathbb{Z}^{+}$and let $\preceq$ be a partial order on $\{1,2, \ldots, n\}$ consistent with the natural order in the sense that $i \preceq j \Rightarrow i \leq j$. Call a subset $\mathcal{F}$ of $\{1,2, \ldots, n\}$ $\preceq$-hereditary (or simply hereditary if no confusion can arise) if $i \preceq j \in \mathcal{F} \Rightarrow i \in \mathcal{F}$. The union and the intersection of hereditary subsets are again hereditary, so the set of hereditary subsets is a finite distributive lattice under the inclusion ordering. We will denote this lattice by $\mathcal{D}_{n}(\preceq)$. The non-zero join-irreducible elements of $\mathcal{D}_{n}(\preceq)$ are the subsets $\{i: 1 \leq i \leq n$ and $i \preceq j\}, 1 \leq j \leq n$. In particular, $\mathcal{D}_{n}(\preceq)$ has height $n$.

If $\left\{H_{i}: 1 \leq i \leq n\right\}$ is a pairwise orthogonal family of non-zero subspaces of the complex separable Hilbert space $H$ satisfying $H=\bigoplus_{i=1}^{n} H_{i}$, the set of subspaces $\left\{\bigoplus_{i \in \mathcal{F}} H_{i}: \mathcal{F} \in \mathcal{D}_{n}(\preceq)\right\}$ is a finite CSL on the Hilbert space $H$, lattice-isomorphic to $\mathcal{D}_{n}(\preceq)$ by the map $\mathcal{F} \mapsto \bigoplus_{i \in \mathcal{F}} H_{i}$. All finite CSL's on separable Hilbert space arise in this way. Indeed, let $\mathcal{L}$ be a finite CSL on the Hilbert space $H$. Then, as is well known, $\mathcal{L}$ is distributive. Let $\left\{K_{j}: 1 \leq j \leq n\right\}$ be the set of non-zero join-irreducible elements of $\mathcal{L}$, enumerated so that $K_{i} \subseteq K_{j} \Rightarrow i \leq j$, and define the partial order $\preceq$ on $\{1,2, \ldots, n\}$ by $i \preceq j$ if $K_{i} \subseteq K_{j}$. Then $\preceq$ is consistent with the natural order. For each $1 \leq i \leq n, K_{i} \cap\left(K_{i}\right)_{-}$is the unique element of $\mathcal{L}$ covered by $K_{i}$. Put $H_{i}=K_{i} \ominus\left(K_{i} \cap\left(K_{i}\right)_{-}\right), 1 \leq i \leq n$. Then $H_{i} \perp H_{j}$ if $i \neq j$. For, if $i \neq j$ and $K_{i} \subseteq K_{j}$, then $K_{i} \subseteq K_{j} \cap\left(K_{j}\right)_{-}$so $H_{j} \subseteq\left(K_{j} \cap\left(K_{j}\right)_{-}\right)^{\perp} \subseteq K_{i}^{\perp} \subseteq H_{i}^{\perp}$. On the other hand, if $K_{i} \nsubseteq K_{j}$, then $K_{j} \subseteq\left(K_{i}\right)_{-}$so $H_{i} \cap H_{j} \subseteq K_{i} \cap K_{j} \subseteq K_{i} \cap\left(K_{i}\right)_{-}$. Thus $H_{i} \cap H_{j} \subseteq\left(K_{i} \cap\left(K_{i}\right)_{-}\right)^{\perp} \cap\left(K_{i} \cap\left(K_{i}\right)_{-}\right)$so $H_{i} \cap H_{j}=(0)$. Since the projections $P_{H_{i}}$ and $P_{H_{j}}$ commute, it follows that $H_{i} \perp H_{j}$. Continuing, we also have $K_{j}=\bigoplus\left\{H_{i}: H_{i} \subseteq K_{j}\right\}$, for every $1 \leq j \leq n$. This can be proved by induction on the height of $K_{j}$ in $\mathcal{L}$. Note that if the height of $K_{j}$ is 1 , then $K_{j}$ is an atom of $\mathcal{L}$ and $K_{j} \cap\left(K_{j}\right)_{-}=(0)$, so $K_{j}=H_{j}$. For every $1 \leq j \leq n, K_{j}=$ $H_{j} \oplus\left(K_{j} \cap\left(K_{j}\right)_{-}\right)$, where the height of $K_{j} \cap\left(K_{j}\right)_{-}$is strictly less than the height of $K_{j}$. Since $K_{j} \cap\left(K_{j}\right)_{-}$is the closed linear span of non-zero join-irreducible elements each of height strictly less than that of $K_{j}$, it follows by the induction assumption that $K_{j}=\bigoplus\left\{H_{i}: H_{i} \subseteq K_{j}\right\}$. Since $H=\bigvee_{j=1}^{n} K_{j}$, we have $H=\bigoplus_{j=1}^{n} H_{j}$. Also, since $H_{i} \subseteq K_{j}$ if and only if $K_{i} \subseteq K_{j}\left(H_{i} \subseteq K_{j}\right.$ and $K_{i} \nsubseteq K_{j}$ give $K_{j} \subseteq\left(K_{i}\right)_{-}$ and so $\left.H_{i} \subseteq K_{i} \cap\left(K_{i}\right)_{-}\right), K_{j}=\bigoplus\left\{H_{i}: K_{i} \subseteq K_{j}\right\}$, for every $1 \leq j \leq n$. More generally, since $L=\bigvee\left\{K_{j}: K_{j} \subseteq L\right\}$, for every $L \in \mathcal{L}$, we have $L=\bigoplus\left\{H_{j}\right.$ : $\left.K_{j} \subseteq L\right\}$. Clearly $\left\{j: K_{j} \subseteq L\right\}$ is $\preceq$-hereditary. Finally, note that for every $\preceq$-hereditary subset $\mathcal{F}$ of $\{1,2, \ldots, n\}$ we have $\bigoplus\left\{H_{j}: j \in \mathcal{F}\right\}=\bigvee\left\{K_{j}: j \in \mathcal{F}\right\}$ (if $j \in \mathcal{F}$, then $K_{j}=\bigoplus\left\{H_{i}: K_{i} \subseteq K_{j}\right\}$ and $K_{i} \subseteq K_{j}$ implies that $i \in \mathcal{F}$ ). Thus $\bigoplus\left\{H_{j}: j \in \mathcal{F}\right\} \in \mathcal{L}$.

In this way we see that the 'building blocks' of finite CSL's are

(i) an orthogonal decomposition $H=\bigoplus_{i=1}^{n} H_{i}$ and

(ii) a partial order $\preceq$ on $\{1,2, \ldots, n\}$, consistent with the natural order.

With notation as in the preceding paragraph, the non-zero join-irreducible elements of $\mathcal{L}$ are the elements $\bigoplus\left\{H_{i}: i \preceq j\right\}, 1 \leq j \leq n$. An operator $T \in \mathcal{B}(H)$ belongs to $\operatorname{Alg} \mathcal{L}$ if and only if it leaves each of the subspaces $\bigoplus\left\{H_{i}: i \preceq j\right\}$ 
invariant; equivalently, the $n \times n$ (operator) matrix $\left(T_{i, j}\right)$ of $T$ relative to the decomposition $H=\bigoplus_{i=1}^{n} H_{i}$ satisfies $T_{i, j}=0$ whenever $i \npreceq j$. Thus we may write

$$
\operatorname{Alg} \mathcal{L}=\left\{\left(T_{i, j}\right) \in \mathcal{B}(H): T_{i, j} \in \mathcal{B}\left(H_{j}, H_{i}\right) \text { and } T_{i, j}=0 \text { if } i \npreceq j\right\} .
$$

The requirement that $\preceq$ be consistent with the natural order can be dropped from the preceding three paragraphs. The reason that we have included it, and will continue to do so, is that, with notation as in the preceding paragraph, Alg $\mathcal{L}$ becomes an algebra of upper-triangular operator-entried matrices when $\preceq$ is consistent with the natural order.

An element $s$ of an (abstract) algebra $\mathcal{A}$ is a single element of $\mathcal{A}$ if $a s b=0$ and $a, b \in \mathcal{A}$ imply that $a s=0$ or $s b=0$. (This notion is useful, for example, in the representation theory of normed algebras [3.) If $\left\{u_{1}, u_{2}, \ldots, u_{n}\right\}$ is the usual orthonormal basis for $\mathbb{F}^{n}$ and $\preceq$ is a partial order on $\{1,2, \ldots, n\}$ consistent with the natural order, then

$$
\mathcal{L}_{n}(\preceq)=\left\{\bigvee\left\{u_{i}: i \in \mathcal{F}\right\}: \mathcal{F} \text { is a } \preceq \text {-hereditary subset of }\{1,2, \ldots, n\}\right\}
$$

is a finite CSL on $\mathbb{F}^{n}$ and $\operatorname{Alg} \mathcal{L}_{n}(\preceq)$ can be identified with the matrix incidence algebra

$$
\mathcal{A}_{n}(\preceq)=\left\{\left(a_{i, j}\right) \in M_{n}(\mathbb{F}): a_{i, j}=0 \text { if } i \npreceq j\right\} .
$$

The single elements of $\mathcal{A}_{n}(\preceq)$ have been described, in terms of the partial order $\preceq$, in [7] (see Theorem 1 below). We show below how this leads to a description of the single elements of $\operatorname{Alg} \mathcal{L}$, for any finite CSL $\mathcal{L}$. Actually, our main result applies in a slightly wider context. Let $\mathcal{D}$ be a finite distributive subspace lattice on $H$ with the property that the vector sum $K+L$ is closed, for every $K, L \in \mathcal{D}$. (This would be the case if, for example, $\operatorname{dim} H<\infty$.) Let the set of non-zero join-irreducible elements of $\mathcal{D}$ be $\left\{K_{j}: 1 \leq j \leq n\right\}$, enumerated so that $K_{i} \subseteq K_{j} \Rightarrow i \leq j$. Then, as in the commutative case, we can define $H_{i}=K_{i} \ominus\left(K_{i} \cap\left(K_{i}\right)_{-}\right)$and it turns out that (see the proof of [4, Theorem 8]) $H=\sum_{i=1}^{n} H_{i}$ where the $H_{i}$ 's are independent (but not necessarily pairwise orthogonal) in the sense that $H_{i} \cap\left(\sum_{j \neq i} H_{j}\right)=(0)$. Moreover,

$$
\mathcal{D}=\left\{\sum_{j \in \mathcal{F}} H_{j}: \mathcal{F} \text { is a } \preceq \text {-hereditary subset of }\{1,2, \ldots, n\}\right\},
$$

where again $\preceq$ is defined by $i \preceq j$ if $K_{i} \subseteq K_{j}$. Conversely, every such independent decomposition $H=\sum_{i=1}^{n} H_{i}$ of $H$ and consistent partial order $\preceq$ on $\{1,2, \ldots, n\}$ gives rise to a finite distributive subspace lattice $\mathcal{D}$ on $H$, defined by $(*)$ with the property that $K+L$ is closed, for every $K, L \in \mathcal{D}$. Of course, there is a natural matrix representation of $\operatorname{Alg} \mathcal{D}$ for such a subspace lattice $\mathcal{D}$, as in the commutative case. We will not pursue this further as there is nothing essentially different here from the commutative case. Indeed, by [4, Corollary 7.1, Theorem 8] such a finite distributive subspace lattice is similar to a finite CSL.

Given a finite distributive subspace lattice $\mathcal{D}$ on a real or complex Banach space, with the property that the vector sum $K+L$ is closed, for every $K, L \in \mathcal{D}$, the expression $K_{i} \ominus\left(K_{i} \cap\left(K_{i}\right)_{-}\right)$will usually make no sense (with $\left(K_{i}\right)_{-}$calculated in $\mathcal{D}$, as earlier). So the subspace 'blocks' with which the representation of $\mathcal{D}$ may be built are not immediately apparent. However, note that if the underlying space is a Hilbert space, and $H_{i}=K_{i} \ominus\left(K_{i} \cap\left(K_{i}\right)_{-}\right), 1 \leq i \leq n$, then $\left\{H_{i}\right.$ : $1 \leq i \leq n\}$ is the set of atoms of an ABSL $\mathcal{B}$ on $H$ satisfying $\mathcal{D} \subseteq \mathcal{B}$. Moreover, 
$H_{i}=K_{i} \cap\left(K_{i} \cap\left(K_{i}\right)_{-}\right)^{\prime}$ where $^{\prime}$ denotes Boolean complement (taken in $\left.\mathcal{B}\right)$. For, $H_{i} \cap\left(K_{i} \cap\left(K_{i}\right)_{-}\right)=(0)$ so $H_{i} \subseteq\left(K_{i} \cap\left(K_{i}\right)_{-}\right)^{\prime}$. On the other hand, if $H_{j} \subseteq$ $K_{i} \cap\left(K_{i} \cap\left(K_{i}\right)_{-}\right)^{\prime}$, then $H_{j} \nsubseteq K_{i} \cap\left(K_{i}\right)_{-}$and $H_{j} \subseteq K_{i}$, so $H_{j} \nsubseteq\left(K_{i}\right)_{-}$. Thus $K_{j} \nsubseteq\left(K_{i}\right)_{-}$so $K_{i} \subseteq K_{j}$. Also, $K_{j} \subseteq K_{i}$ (since otherwise $H_{j} \subseteq K_{i} \subseteq\left(K_{j}\right)_{-}$) so $i=j$.

This suggests the class of finite distributive subspace lattices with which the present note is primarily concerned. Namely, those finite distributive subspace lattices $\mathcal{D}$ on $X$ for which there exists a finite ABSL $\mathcal{B}$ on $X$ with the property that the vector sum $K+L$ is closed, for every $K, L \in \mathcal{B}$, satisfying $\mathcal{D} \subseteq \mathcal{B}$. It is for such distributive subspace lattices that we describe the single elements of $\operatorname{Alg} \mathcal{D}$ below (Theorem 2). If the underlying space is a separable complex Hilbert space $H$, such finite distributive subspace lattices are precisely those that are similar to a finite CSL [4, Corollary 7.1]. If $\operatorname{dim} H<\infty$, every distributive subspace lattice on $H$ is of this type [4. Corollaries 7.1, 8.1].

\section{MAIN THEOREM}

Throughout the remainder of this note we let $\mathcal{D}$ be a finite distributive subspace lattice on $X$ and let $\mathcal{B}$ be a finite ABSL on $X$ such that $\mathcal{D} \subseteq \mathcal{B}$ and such that the vector sum $K+L$ is closed, for every $K, L \in \mathcal{B}$. Let $\left\{L_{1}, L_{2}, \ldots, L_{m}\right\}$ be the set of atoms of $\mathcal{B}$. Let $\left\{K_{1}, K_{2}, \ldots, K_{n}\right\}$ be the set of non-zero join-irreducible elements of $\mathcal{D}$ enumerated so that $K_{i} \subseteq K_{j}$ implies that $i \leq j$. Let $\preceq$ be the partial order on $\{1,2, \ldots, n\}$ defined by $i \preceq j$ if $K_{i} \subseteq K_{j}$. For each $1 \leq i \leq n$ put $W_{i}=K_{i} \cap\left(K_{i} \cap\left(K_{i}\right)_{-}\right)^{\prime}$, where ' denotes Boolean complementation in $\mathcal{B}$, and $\left(K_{i}\right)_{-}$is calculated in $\mathcal{D}$. Then, $W_{i} \in \mathcal{B}, 1 \leq i \leq n$. Our main theorem is to be used in conjunction with the following description of the single elements of $\mathcal{A}_{n}(\preceq)$, which we include for the convenience of the reader.

Theorem 1 ([7]). Let $\mathcal{A}_{n}(\preceq)$ be a matrix incidence algebra over a field $K$. The matrix $S \in \mathcal{A}_{n}(\preceq)$ is a single element of $\mathcal{A}_{n}(\preceq)$ if and only if

(i) $r_{i} \neq 0$ and $c_{j} \neq 0 \quad(1 \leq i, j \leq n) \Rightarrow s_{i, j} \neq 0$,

(ii) $i \preceq j_{1}$ and $i \preceq j_{2}$, for some $1 \leq i \leq n \Rightarrow r_{j_{1}}$ and $r_{j_{2}}$ are linearly dependent, dependent.

(iii) $i_{1} \preceq j$ and $i_{2} \preceq j$, for some $1 \leq j \leq n \Rightarrow c_{i_{1}}$ and $c_{i_{2}}$ are linearly

Here $r_{i}$ and $c_{j}$ denote the $i$-th row and the $j$-th column of $S$, respectively.

Before proving our main theorem it is convenient to make some observations.

Observations. 1 . From the fact that $K+L$ is closed, for every $K, L \in \mathcal{B}$ it readily follows that, for every finite set $\left\{M_{k}: 1 \leq k \leq p\right\}$ of elements of $\mathcal{B}, \sum_{k=1}^{p} M_{k}$ is a closed vector sum (equal to $\bigvee_{k=1}^{p} M_{k}$ ).

2. Each $W_{i}$ is the vector sum of a unique subset of $\left\{L_{k}: 1 \leq k \leq m\right\}$. This subset is non-empty since $W_{i} \neq(0)$. (Observe that $W_{i} \vee\left(K_{i} \cap\left(K_{i}\right)_{-}\right)=K_{i}$ and $\left.W_{i} \cap\left(K_{i} \cap\left(K_{i}\right)_{-}\right)=(0).\right)$

3. If $i \neq j$, the subsets of $\left\{L_{k}: 1 \leq k \leq m\right\}$ comprising $W_{i}$ and $W_{j}$ are disjoint. This is because $W_{i} \cap W_{j}=(0)$. For, if $i \neq j$, then $K_{i} \neq K_{j}$ so, without loss of generality, $K_{i} \nsubseteq K_{j}$. Then $K_{j} \subseteq\left(K_{i}\right)_{-}$, so

$$
W_{i} \cap W_{j} \subseteq\left(K_{i} \cap K_{j}\right) \cap\left(K_{i} \cap\left(K_{i}\right)_{-}\right)^{\prime} \subseteq\left(K_{i} \cap\left(K_{i}\right)_{-}\right) \cap\left(K_{i} \cap\left(K_{i}\right)_{-}\right)^{\prime}=(0) .
$$


4. For every $1 \leq j \leq n, K_{j}=\bigvee\left\{W_{i}: W_{i} \subseteq K_{j}\right\}$. This can be proved by induction on the height of $K_{j}$ in $\mathcal{D}$. If the height of $K_{j}$ is 1 , then $K_{j}$ is an atom of $\mathcal{D}$. Then $K_{j} \cap\left(K_{j}\right)_{-}=(0)$, so $K_{j}=W_{j}$. Since $W_{i} \cap W_{j}=(0)$ if $i \neq j$, $K_{j}=\bigvee\left\{W_{i}: W_{i} \subseteq K_{j}\right\}$. To employ the induction assumption observe that, for every $1 \leq j \leq n, K_{j}=W_{j} \vee\left(K_{j} \cap\left(K_{j}\right)_{-}\right)$where the height of $K_{j} \cap\left(K_{j}\right)_{-}$is strictly less than that of $K_{j}$.

5. Since $\bigvee_{i=1}^{n} K_{i}=X$ we have $\bigvee_{i=1}^{n} W_{i}=X$. It follows that the subsets of $\left\{L_{1}, L_{2}, \ldots, L_{m}\right\}$ which comprise the $W_{i}$ 's form a partition of $\left\{L_{1}, L_{2}, \ldots, L_{m}\right\}$. Thus (see [1, Example 2.7(1)(ii)]) $\left\{W_{1}, W_{2}, \ldots, W_{n}\right\}$ is the set of atoms of a finite ABSL on $X, \mathcal{C}$ say, satisfying $\mathcal{D} \subseteq \mathcal{C} \subseteq \mathcal{B}$. It is also clear that the vector sum $K+L$ is closed, for every $K, L \in \mathcal{C}$, and that the Boolean complement appearing in the definition of $W_{i}$ can be considered to be taken in $\mathcal{C}$. In short, we may suppose at the outset that $m=n$ (that is, that $\mathcal{C}=\mathcal{B}$ ). We will assume this in what follows.

6. For every $1 \leq j \leq n, K_{j}=\bigvee_{i \prec j} W_{i}$. To prove this it is enough, in view of observation 4 above, to show that $W_{i} \subseteq K_{j} \Leftrightarrow K_{i} \subseteq K_{j}$. The backward implication is obvious. Suppose that $W_{i} \subseteq K_{j}$ and $K_{i} \nsubseteq K_{j}$. Then $K_{j} \subseteq\left(K_{i}\right)_{-}$, so

$$
W_{i} \subseteq\left(K_{i} \cap\left(K_{i}\right)_{-}\right) \cap\left(K_{i} \cap\left(K_{i}\right)_{-}\right)^{\prime}=(0),
$$

which contradicts $W_{i} \neq(0)$. Thus $W_{i} \subseteq K_{j} \Rightarrow K_{i} \subseteq K_{j}$.

7. For every $\preceq$-hereditary subset $\mathcal{F}$ of $\{1,2, \ldots, n\}$ we have $\bigvee\left\{W_{j}: j \in \mathcal{F}\right\}=$ $\bigvee\left\{K_{j}: j \in \mathcal{F}\right\}$. For, if $j \in \mathcal{F}$, then $K_{j} \subseteq \bigvee\left\{W_{i}: i \in \mathcal{F}\right\}$ using the preceding observation. Hence $\bigvee\left\{K_{j}: j \in \mathcal{F}\right\} \subseteq \bigvee\left\{W_{i}: i \in \mathcal{F}\right\}$, and the reverse inclusion is obvious. For every $L \in \mathcal{D}, L=\bigvee\left\{K_{j}: K_{j} \subseteq L\right\}$, where $\left\{j: K_{j} \subseteq L\right\}$ is hereditary. Thus $L=\bigvee\left\{W_{j}: K_{j} \subseteq L\right\}$. This shows that the map $\phi: \mathcal{D}_{n}(\preceq) \rightarrow \mathcal{D}$ defined by $\phi(\mathcal{F})=\bigvee\left\{W_{j}: j \in \mathcal{F}\right\}$ is surjective. If $\mathcal{F}, \mathcal{G}$ are hereditary subsets of $\{1,2, \ldots, n\}$, then $\mathcal{F} \subseteq \mathcal{G} \Leftrightarrow \phi(\mathcal{F}) \subseteq \phi(\mathcal{G})$ (the reverse implication follows easily from the fact that $\left\{W_{1}, W_{2}, \ldots, W_{n}\right\}$ is the set of atoms of an ABSL). Thus $\phi$ is a lattice-isomorphism. In particular, $\left(K_{j}\right)_{-}=\bigvee_{j \npreceq i} W_{i}=\left(\bigvee_{j \preceq i} W_{i}\right)^{\prime}=\bigcap_{j \preceq i} W_{i}^{\prime}$.

8. By 11 Theorem 2.8], $\mathcal{B}^{\perp}=\left\{M^{\perp}: M \in \mathcal{B}\right\}$ is an ABSL on $X^{*}$ with set of atoms $\left\{\left(W_{1}^{\prime}\right)^{\perp},\left(W_{2}^{\prime}\right)^{\perp}, \ldots,\left(W_{n}^{\prime}\right)^{\perp}\right\}$. The vector sum $K+L$ is closed, for every $K, L \in \mathcal{B}^{\perp}$. For, each $x \in X$ has a unique decomposition $x=\sum_{i=1}^{n} x_{i}$ with $x_{i} \in W_{i}, 1 \leq i \leq n$, and the map $P_{i}: X \rightarrow X$ defined by $P_{i} x=x_{i}$ is linear and closed, and so is bounded by the Closed Graph Theorem. Clearly $\left(W_{j}^{\prime}\right)^{\perp} \subseteq \operatorname{ker} P_{i}^{*}$ if $i \neq j$ and $\mathcal{R}\left(P_{i}^{*}\right) \subseteq\left(W_{i}^{\prime}\right)^{\perp}$, for $1 \leq i \leq n$ (where, for any operator $T, \mathcal{R}(T)$ denotes the range of $T)$. In fact $\mathcal{R}\left(P_{i}^{*}\right)=\left(W_{i}^{\prime}\right)^{\perp}$, since $P_{i}^{*} e^{*}=e^{*}$, for every $e^{*} \in\left(W_{i}^{\prime}\right)^{\perp}$. Thus $P_{i}^{*}\left(\sum_{j=1}^{n} e_{j}^{*}\right)=e_{i}^{*}$ whenever $e_{j}^{*} \in\left(W_{j}^{\prime}\right)^{\perp}, 1 \leq j \leq n$. The continuity of the $P_{i}^{*}$ now shows that $\bigvee_{j \in \mathcal{E}}\left(W_{j}^{\prime}\right)^{\perp}=\sum_{j \in \mathcal{E}}\left(W_{j}^{\prime}\right)^{\perp}$, for every subset $\mathcal{E}$ of $\{1,2, \ldots, n\}$ and it follows that $K+L$ is closed, for every $K, L \in \mathcal{B}^{\perp}$. Hence each $y^{*} \in X^{*}$ has a unique decomposition $y^{*}=\sum_{j=1}^{n} y_{j}^{*}$ with $y_{j}^{*} \in\left(W_{j}^{\prime}\right)^{\perp}, 1 \leq j \leq n$.

9. By [5, Lemmas 2.1, 3.1] it readily follows that an element $S \in \operatorname{Alg} \mathcal{D}$ is a single element if and only if whenever $g^{*} \in X^{*}, h \in X$ are vectors for which elements $J, K \in \mathcal{D}$ exist, satisfying $h \in J, g^{*} \in\left(K_{-}\right)^{\perp}, J_{-} \neq X, K \neq(0)$, and $g^{*}(S h)=0$, then $S h=0$ or $S^{*} g^{*}=0$.

Theorem 2. The operator $S \in \mathcal{B}(X)$ is a single element of $A l g \mathcal{D}$ if and only if there exist non-zero vectors $f_{i} \in W_{i}, e_{j}^{*} \in\left(W_{j}^{\prime}\right)^{\perp}, 1 \leq i, j \leq n$, and a single element $\left(s_{i, j}\right)$ of $\mathcal{A}_{n}(\preceq)$ such that $S=\sum_{i=1}^{n} \sum_{j=1}^{n} s_{i, j}\left(e_{j}^{*} \otimes f_{i}\right)$. Moreover, $S$ and $\left(s_{i, j}\right)$ have the same rank. 
Proof. Let $\left(s_{i, j}\right) \in \mathcal{A}_{n}(\preceq)$ and let $f_{i} \in W_{i}, e_{j}^{*} \in\left(W_{j}^{\prime}\right)^{\perp}, 1 \leq i, j \leq n$, be arbitrary vectors. We show that the operator

$$
S=\sum_{i=1}^{n} \sum_{j=1}^{n} s_{i, j}\left(e_{j}^{*} \otimes f_{i}\right)
$$

belongs to $\operatorname{Alg} \mathcal{D}$. For this it is enough to show that $S K_{k} \subseteq K_{k}$, for every $1 \leq k \leq n$. If $j \npreceq k$, then $K_{k}=\bigvee_{l \preceq k} W_{l} \subseteq W_{j}^{\prime}$, so $e_{j}^{*} \in\left(K_{k}\right)^{\perp}$. Thus, for every $x \in K_{k}$,

$$
S x=\sum_{i=1}^{n}\left(\sum_{j \preceq k} s_{i, j} e_{j}^{*}(x)\right) f_{i} .
$$

But $s_{i, j}=0$ if $i \npreceq j$, so

$$
S x=\sum_{i \preceq k}\left(\sum_{j \preceq k} s_{i, j} e_{j}^{*}(x)\right) f_{i} \in \bigvee_{i \preceq k} W_{i}=K_{k} .
$$

Thus $S \in \operatorname{Alg} \mathcal{D}$.

Now, additionally, let $\left(s_{i, j}\right)$ be a single element of $\mathcal{A}_{n}(\preceq)$ and let $f_{i}, e_{j}^{*}, 1 \leq$ $i, j \leq n$, be non-zero vectors. We show that $S$ is a single element of Alg $\mathcal{D}$. By observation 9 above it is enough to show that if $h \in X, g^{*} \in X^{*}$ and $g^{*}(S h)=0$ and there exist elements $J, K \in \mathcal{D}$ such that $h \in J, g^{*} \in\left(K_{-}\right)^{\perp}$ and $J_{-} \neq X, K \neq(0)$, then $S h=0$ or $S^{*} g^{*}=0$. Let $g^{*}(S h)=0$. Now $g^{*}(S h)=\sum_{i, j=1}^{n} s_{i, j} e_{j}^{*}(h) g^{*}\left(f_{i}\right)$. Put $\tilde{g}=\sum_{i=1}^{n} \overline{g^{*}\left(f_{i}\right)} u_{i}$ and $\tilde{h}=\sum_{j=1}^{n} e_{j}^{*}(h) u_{j}$ (where $\left\{u_{1}, u_{2}, \ldots, u_{n}\right\}$ is the usual orthonormal basis for $\left.\mathbb{F}^{n}\right)$. Then $\tilde{g}, \tilde{h} \in \mathbb{F}^{n}$ and $g^{*}(S h)=(\tilde{S} \tilde{h} \mid \tilde{g})$, where $\tilde{S}=\left(s_{i, j}\right)$ is regarded as an operator on $\mathbb{F}^{n}$ (and $(\cdot \mid \cdot)$ denotes the usual inner-product on $\left.\mathbb{F}^{n}\right)$. Now $K_{-}=\bigvee_{k \in \mathcal{E}} W_{k}$ for some hereditary subset $\mathcal{E}$ of $\{1,2, \ldots, n\}$. It follows that there exists $\tilde{K} \in \mathcal{L}_{n}(\preceq)$, with $\tilde{K} \neq(0)$, such that $(\tilde{K})_{-}=\bigvee_{k \in \mathcal{E}} u_{k}$ (the map $\bigvee_{k \in \mathcal{F}} W_{k} \mapsto \bigvee_{k \in \mathcal{F}} u_{k}$, with $\mathcal{F}$ hereditary, is a lattice-isomorphism of $\mathcal{D}$ onto $\left.\mathcal{L}_{n}(\preceq)\right)$. If $k \in \mathcal{E}$, then $W_{k} \subseteq K_{-}$, so $f_{k} \in K_{-}$and $g^{*}\left(f_{k}\right)=0=\left(\tilde{g} \mid u_{k}\right)$. Hence $\tilde{g} \in\left((\tilde{K})_{-}\right)^{\perp}$. Similarly, there exists $\tilde{J} \in \mathcal{L}_{n}(\preceq)$, with $(\tilde{J})_{-} \neq \mathbb{F}^{n}$, such that $\tilde{h} \in \tilde{J}$. So, again by observation 9 above, since $\tilde{S}$ is single and $(\tilde{S} \tilde{h} \mid \tilde{g})=0$, we have $\tilde{S} \tilde{h}=0$ or $(\tilde{S})^{*} \tilde{g}=0$. In the former case $S h=0$ and in the latter $S^{*} g^{*}=0$. (Note that in this part of the proof we did not use the fact that all of the vectors $f_{i} \in W_{i}, e_{j}^{*} \in\left(W_{j}^{\prime}\right)^{\perp}, 1 \leq i, j \leq n$, are non-zero.)

Conversely, let $S$ be a single element of Alg $\mathcal{D}$. For every $1 \leq j \leq n, W_{j} \subseteq K_{j}$ and, by [5. Lemma 3.2], $S K_{j}$ is at most one-dimensional. Hence $S W_{j}$ is at most one-dimensional. Let $S W_{j}=\left\langle g_{j}\right\rangle$ with $S c_{j}=g_{j}, c_{j} \in W_{j}$ and let $g_{j}=\sum_{k=1}^{n} f_{k, j}$ with $f_{k, j} \in W_{k}, 1 \leq k \leq n$, be the unique decomposition of $g_{j}$. (Here, and in what follows, we let $\langle x\rangle$ denote the linear span of $\{x\}$, for every vector $x$.) Similarly, since $\left(W_{i}^{\prime}\right)^{\perp} \subseteq\left(\left(K_{i}\right)_{-}\right)^{\perp}$, for every $1 \leq i \leq n$ (see observation 7$)$, and $S^{*}\left(\left(K_{i}\right)_{-}\right)^{\perp}$ is at most one-dimensional [5, Lemma 3.3], $S^{*}\left(W_{i}^{\prime}\right)^{\perp}=\left\langle h_{i}^{*}\right\rangle$ with $S^{*} d_{i}^{*}=h_{i}^{*}, d_{i}^{*} \in\left(W_{i}^{\prime}\right)^{\perp}$ and $h_{i}^{*}=\sum_{l=1}^{n} e_{i, l}^{*}, e_{i, l}^{*} \in\left(W_{l}^{\prime}\right)^{\perp}, 1 \leq l \leq n$, the unique decomposition of $h_{i}^{*}$ (see observation 8 ).

First we show that if $f_{i, j} \neq 0$ and $f_{i, k} \neq 0$, then $\left\langle f_{i, j}\right\rangle=\left\langle f_{i, k}\right\rangle$. Now $f_{i, j} \neq 0$ gives $g_{j} \notin W_{i}^{\prime}$ so there exists $e^{*} \in\left(W_{i}^{\prime}\right)^{\perp}$ such that $e^{*}\left(g_{j}\right) \neq 0$. Then $S^{*} e^{*} \neq 0$ since $S^{*} e^{*}\left(c_{j}\right)=e^{*}\left(g_{j}\right)$. Thus $S^{*}\left(W_{i}^{\prime}\right)^{\perp} \neq(0)$ and $h_{i}^{*} \neq 0$. For every $x^{*} \in\left(W_{i}^{\prime}\right)^{\perp}$ there exists a unique scalar $\lambda$ such that $S^{*} x^{*}=\lambda h_{i}^{*}$. Then

$$
x^{*}\left(g_{j}\right)=x^{*}\left(f_{i, j}\right)=x^{*}\left(S c_{j}\right)=\lambda h_{i}^{*}\left(c_{j}\right)=\lambda e_{i, j}^{*}\left(c_{j}\right) .
$$


Taking $x^{*}$ equal to $e^{*}$ shows that $e_{i, j}^{*}\left(c_{j}\right) \neq 0$. Similarly, $e_{i, k}^{*}\left(c_{k}\right) \neq 0$. If $f_{i, j}$ and $f_{i, k}$ were linearly independent there would exist $y^{*} \in\left(W_{i}^{\prime}\right)^{\perp}$ such that $y^{*}\left(f_{i, j}\right)=0$ and $y^{*}\left(f_{i, k}\right) \neq 0$ (there would exist a bounded linear functional $y^{*}$ on $W_{i}$ such that $y^{*}\left(f_{i, j}\right)=0$ and $y^{*}\left(f_{i, k}\right) \neq 0$. Now extend $y^{*}$ to a bounded linear functional on $X$ by defining $y^{*}(z)=y^{*}\left(P_{i} z\right), z \in X$; here $P_{i}$ is as in observation 8). If $S^{*} y^{*}=\mu h_{i}^{*}$, then $y^{*}\left(f_{i, j}\right)=\mu e_{i, j}^{*}\left(c_{j}\right)=0$ and $y^{*}\left(f_{i, k}\right)=\mu e_{i, k}^{*}\left(c_{k}\right) \neq 0$, which is clearly a contradiction. Thus $\left\langle f_{i, j}\right\rangle=\left\langle f_{i, k}\right\rangle$.

A similar proof shows that $e_{i, j}^{*} \neq 0$ and $e_{k, j}^{*} \neq 0$ imply that $\left\langle e_{i, j}^{*}\right\rangle=\left\langle e_{k, j}^{*}\right\rangle$. (Note that $e_{i, j}^{*} \neq 0$ implies that $h_{i}^{*} \notin W_{j}^{\perp}$ so $S W_{j} \neq(0)$ and $g_{j} \neq 0$. For every $z \in W_{j}$ there exists $\gamma$ such that $e_{i, j}^{*}(z)=\gamma d_{i}^{*}\left(f_{i, j}\right)$ and $e_{k, j}^{*}(z)=\gamma d_{k}^{*}\left(f_{k, j}\right)$ with $d_{i}^{*}\left(f_{i, j}\right) \neq 0$ and $d_{k}^{*}\left(f_{k, j}\right) \neq 0$. If $e_{i, j}^{*}$ and $e_{k, j}^{*}$ were linearly independent, there would exist $z \in W_{j}$ such that $e_{i, j}^{*}(z)=0$ and $e_{k, j}^{*}(z) \neq 0$. Contradiction.)

By considering the arrays of vectors $\left(f_{i, j}\right),\left(e_{i, j}^{*}\right)$ it now follows that there exist non-zero vectors $f_{i}, e_{j}^{*}, 1 \leq i, j \leq n$, with $f_{i} \in W_{i}, e_{j}^{*} \in\left(W_{j}^{\prime}\right)^{\perp}$ and scalars $\lambda_{i, j}, \mu_{i, j}, 1 \leq i, j \leq n$, such that $f_{i, j}=\lambda_{i, j} f_{i}, e_{i, j}^{*}=\mu_{i, j} e_{j}^{*}$, for all $i$ and $j$. Note that since $d_{i}^{*}\left(g_{j}\right)=h_{i}^{*}\left(c_{j}\right)$, for every $i$ and $j, d_{i}^{*}\left(g_{j}\right) \neq 0$ implies that $g_{j} \neq 0$ and $h_{i}^{*} \neq 0$. The reverse implication also holds since $S$ is single. (Note that $c_{j} \in K_{j}$ and $d_{i}^{*} \in\left(\left(K_{i}\right)_{-}\right)^{\perp}$. If $d_{i}^{*}\left(g_{j}\right)=0$, then $d_{i}^{*}\left(S c_{j}\right)=0$, whence by singleness, and observation 9 above, $S c_{j}=0$ or $S^{*} d_{i}^{*}=0$.)

Define the $n \times n$ scalar matrix $\left(s_{i, j}\right)$ by

$$
s_{i, j}= \begin{cases}\mu_{i, j} \lambda_{i, j} / d_{i}^{*}\left(g_{j}\right) & \text { if } g_{j} \neq 0 \text { and } h_{i}^{*} \neq 0, \\ 0 & \text { otherwise. }\end{cases}
$$

We show that $S=\sum_{i=1}^{n} \sum_{j=1}^{n} s_{i, j}\left(e_{j}^{*} \otimes f_{i}\right)$. Let $1 \leq i, j \leq n$ and $x \in X$ be arbitrary. Then $x$ has a unique decomposition $x=\sum_{k=1}^{n} x_{k}$, with $x_{k} \in W_{k}, 1 \leq k \leq n$, and there exist scalars $\alpha_{k}, 1 \leq k \leq n$, such that $S x_{k}=\alpha_{k} g_{k}$. Now

$$
h_{i}^{*}\left(x_{j}\right)=d_{i}^{*}\left(S x_{j}\right)=\alpha_{j} d_{i}^{*}\left(g_{j}\right)=\left(\sum_{l=1}^{n} \mu_{i, l} e_{l}^{*}\right)\left(x_{j}\right)=\mu_{i, j} e_{j}^{*}\left(x_{j}\right)=\mu_{i, j} e_{j}^{*}(x) ;
$$

that is, $\alpha_{j} d_{i}^{*}\left(g_{j}\right)=\mu_{i, j} e_{j}^{*}(x)$. Also,

$$
\sum_{i=1}^{n} \sum_{j=1}^{n} s_{i, j}\left(e_{j}^{*} \otimes f_{i}\right)(x)=\sum_{i=1}^{n} \sum_{j=1}^{n} s_{i, j} e_{j}^{*}(x) f_{i}
$$

and

$$
S x=\sum_{j=1}^{n} S x_{j}=\sum_{j=1}^{n} \sum_{i=1}^{n} \alpha_{j} f_{i, j}=\sum_{i=1}^{n} \sum_{j=1}^{n} \alpha_{j} \lambda_{i, j} f_{i} .
$$

However, $s_{i, j} e_{j}^{*}(x)=\alpha_{j} \lambda_{i, j}$. For, if $g_{j} \neq 0$ and $h_{i}^{*} \neq 0$, then

$$
s_{i, j} e_{j}^{*}(x)=\frac{\mu_{i, j} \lambda_{i, j}}{d_{i}^{*}\left(g_{j}\right)} e_{j}^{*}(x)=\alpha_{j} \lambda_{i, j} .
$$

On the other hand, if $g_{j}=0$ or $h_{i}^{*}=0$, then $\lambda_{i, j}=0$ so once again $s_{i, j} e_{j}^{*}(x)=$ $\alpha_{j} \lambda_{i, j}$. (Note that $g_{j}=\sum_{i=1}^{n} \lambda_{i, j} f_{i}$ where $\left\{f_{1}, f_{2}, \ldots, f_{n}\right\}$ is linearly independent. Also, if $h_{i}^{*}=0$, then $S^{*}\left(W_{i}^{\prime}\right)^{\perp}=(0)$ so $\mathcal{R}(S) \subseteq W_{i}^{\prime}, g_{j} \in W_{i}^{\prime}$ and $\lambda_{i, j}=0$.) Thus $S=\sum_{i=1}^{n} \sum_{j=1}^{n} s_{i, j}\left(e_{j}^{*} \otimes f_{i}\right)$. 
Suppose that $s_{i, j} \neq 0$. Then $\lambda_{i, j} \neq 0$ so $g_{j} \notin W_{i}^{\prime}$ and hence (since $g_{j} \in K_{j}$ because $S \in \operatorname{Alg} \mathcal{D}$ ) $K_{j} \nsubseteq\left(K_{i}\right)_{-}=\bigcap_{i \preceq k} W_{k}^{\prime}$ (see observation 7) so $K_{i} \subseteq K_{j}$; that is, $i \preceq j$. This shows that $\left(s_{i, j}\right) \in \mathcal{A}_{n}(\preceq)$.

Next we show that $\left(s_{i, j}\right)$ is a single element of $\mathcal{A}_{n}(\preceq)$. Now, for every $1 \leq$ $i \leq n, f_{i} \notin W_{i}^{\prime}$ so there exists $E_{i}^{*} \in\left(W_{i}^{\prime}\right)^{\perp}$ such that $E_{i}^{*}\left(f_{i}\right)=1$. Also, since $e_{j}^{*} \notin W_{j}^{\perp}$, there exists $F_{j} \in W_{j}$ such that $e_{j}^{*}\left(F_{j}\right)=1$, for every $1 \leq j \leq n$. Clearly $E_{i}^{*}\left(f_{j}\right)=e_{j}^{*}\left(F_{i}\right)=\delta_{i, j}$. Let $A=\left(a_{i, j}\right)$ and $B=\left(b_{i, j}\right)$ be elements of $\mathcal{A}_{n}(\preceq)$ with $A\left(s_{i, j}\right) B=0$. Put $\tilde{A}=\sum_{i=1}^{n} \sum_{j=1}^{n} a_{i, j}\left(E_{j}^{*} \otimes f_{i}\right)$ and $\tilde{B}=\sum_{i=1}^{n} \sum_{j=1}^{n} b_{i, j}\left(e_{j}^{*} \otimes F_{i}\right)$. Then $\tilde{A}, \tilde{B} \in \operatorname{Alg} \mathcal{D}$ (see the first paragraph of the proof of the theorem). We have

$$
\tilde{A} S=\sum_{i, j=1}^{n}\left(\sum_{k=1}^{n} a_{i, k} s_{k, j}\right)\left(e_{j}^{*} \otimes f_{i}\right), \quad S \tilde{B}=\sum_{i, j=1}^{n}\left(\sum_{l=1}^{n} s_{i, l} b_{l, j}\right)\left(e_{j}^{*} \otimes f_{i}\right)
$$

and

$$
\tilde{A} S \tilde{B}=\sum_{i, j=1}^{n}\left(\sum_{k, l=1}^{n} a_{i, k} s_{k, l} b_{l, j}\right)\left(e_{j}^{*} \otimes f_{i}\right) .
$$

Since $\tilde{A} S \tilde{B}=0$ (note that $\sum_{k, l=1}^{n} a_{i, k} s_{k, l} b_{l, j}$ is the $i, j$-th entry of $A\left(s_{i, j}\right) B$ ), and $S$ is single, $\tilde{A} S=0$ or $S \tilde{B}=0$. Since $\left\{e_{j}^{*} \otimes f_{i}: 1 \leq i, j \leq n\right\}$ is a linearly independent family of rank one operators it follows that $A\left(s_{i, j}\right)=0$ or $\left(s_{i, j}\right) B=0$. Hence $\left(s_{i, j}\right)$ is a single element of $\mathcal{A}_{n}(\preceq)$.

Finally we show that $S$ and $\tilde{S}=\left(s_{i, j}\right)$ have the same rank. Note that, if $x \in X$ and $x=\sum_{k=1}^{n} x_{k}$ is the unique decomposition of $x$ with $x_{k} \in W_{k}, 1 \leq k \leq n$, then $e_{j}^{*} \otimes f_{i}(x)=e_{j}^{*}\left(x_{j}\right) f_{i}$ and so $S x=\sum_{i=1}^{n} \lambda_{i} f_{i}$ where $\left(\lambda_{1}, \lambda_{2}, \ldots, \lambda_{n}\right)^{T}=$ $\tilde{S}\left(e_{1}^{*}\left(x_{1}\right), e_{2}^{*}\left(x_{2}\right), \ldots, e_{n}^{*}\left(x_{n}\right)\right)^{T}$. Clearly then $\sum_{i=1}^{n} \mu_{i} f_{i} \in \mathcal{R}(S)$ implies that $\left(\mu_{1}, \mu_{2}\right.$, $\left.\ldots, \mu_{n}\right)^{T} \in \mathcal{R}(\tilde{S})$. The converse is also true. For, with $F_{j} \in W_{j}$ satisfying $e_{j}^{*}\left(F_{j}\right)=$ 1 , if $\left(\mu_{1}, \mu_{2}, \ldots, \mu_{n}\right)^{T}=\tilde{S}\left(\gamma_{1}, \gamma_{2}, \ldots, \gamma_{n}\right)^{T}$, then $S \sum_{k=1}^{n} \gamma_{k} F_{k}=\sum_{i=1}^{n} \mu_{i} f_{i} \in$ $\mathcal{R}(S)$. The map $T: \mathcal{R}(\tilde{S}) \rightarrow \mathcal{R}(S)$ defined by $T\left(\mu_{1}, \mu_{2}, \ldots, \mu_{n}\right)^{T}=\sum_{i=1}^{n} \mu_{i} f_{i}$ is a linear bijection. It follows that $S$ and $\tilde{S}$ have the same rank.

This completes the proof of the theorem.

The preceding theorem shows that, for the type of subspace lattice $\mathcal{D}$ considered, the structure of the single elements of $A \lg \mathcal{D}$ is completely determined by the poset of non-zero join-irreducible elements of $\mathcal{D}$, partially ordered by inclusion. This is consistent with [6] Theorem 3], which shows that the maximum rank that a single element can have is determined by the relationship between the set of maximal elements and the set of minimal elements of this poset. (It is not difficult to show that the non-zero join-irreducible elements occcuring in the unique irredundant representation of $X$ are precisely the maximal ones.)

The preceding theorem can be interpreted matricially. For, by virtue of the decomposition $X=\sum_{i=1}^{n} W_{i}, \mathcal{B}(X)$ may be identified with the set of $n \times n$ operatorentried matrices $\left(T_{i, j}\right)$, with $T_{i, j} \in \mathcal{B}\left(W_{j}, W_{i}\right), 1 \leq i, j \leq n$, and

$$
\operatorname{Alg} \mathcal{D}=\left\{\left(T_{i, j}\right) \in \mathcal{B}(X): T_{i, j} \in \mathcal{B}\left(W_{j}, W_{i}\right) \text { and } T_{i, j}=0 \text { if } i \npreceq j\right\} .
$$

If $f_{i} \in W_{i}$ and $e_{j}^{*} \in\left(W_{j}^{\prime}\right)^{\perp}, 1 \leq i, j \leq n$, are non-zero vectors, then the (operator) matrix $\left(e_{j}^{*} \otimes f_{i}\right)$ belongs to $\mathcal{B}(X)$. The theorem says that the single elements of Alg $\mathcal{D}$ are obtained by taking the Hadamard (that is, entry-wise) product of such 
(operator) matrices with those (scalar) matrices $\left(s_{i, j}\right)$ which are single elements of $\mathcal{A}_{n}(\preceq)$, the rank of the resulting operator being equal to the rank of the latter.

\section{REFERENCES}

[1] S. Argyros, M. Lambrou and W. E. Longstaff, Atomic Boolean subspace lattices and applications to the theory of bases, Memoirs Amer. Math. Soc. 91 (1991). MR 92m:46022

[2] T. Donnellan, Lattice Theory, Pergamon Press, New York, (1968). MR 38:2059

[3] J. A. Erdos, S. Giotopoulos and M. S. Lambrou, Rank one elements of Banach algebras, Mathematika 24 (1977), 178-181. MR 57:7176

[4] K. J. Harrison and W. E. Longstaff, Automorphic images of commutative sub-space lattices, Trans. Amer. Math. Soc. (1) 296 (1986), 217-228. MR 87g:46040

[5] M. S. Lambrou, On the rank of operators in reflexive algebras, Lin. Alg. \& Applic. 142 (1990), 211-235. MR 91k:47104

[6] W. E. Longstaff and Oreste Panaia, On the ranks of single elements of reflexive operator algebras, Proc. Amer. Math. Soc. (10) 125 (1997), 2875-2882. MR 97m:47061

[7] W. E. Longstaff and Oreste Panaia, Single elements of matrix incidence algebras, (manuscript).

Department of Mathematics, The University of Western Australia, Nedlands, Western Australia 6907, Australia

E-mail address: longstaf@maths. uwa.edu.au

Department of Mathematics, The University of Western Australia, Nedlands, Western Australia 6907, Australia

E-mail address: oreste@maths.uwa.edu.au 